\title{
Achieving quality improvement by structured patient management
}

\author{
Martin McNicol
}

It is clear that the quality of the service we give in the NHS needs to be raised. I am confident that it can be raised. In order to do so we have to put aside a long established set of concepts which underlie much of our thinking and which prevent improvement, and we have to come to terms with a fundamental change in health care provision. Only when we have made this change in our thinking will it be possible to achieve the improvement of our services that is increasingly, and rightly, asked of us.

I wish first to consider briefly the outmoded concepts which I believe are still widely prevalent and govern our approach to provision of services. We provide a "take it or leave it" service. Patients are expected to accept what is on offer without question. Delay is inbuilt and is also accepted without question. It may in part be a rationing mechanism, but we impose many more delays than this can justify. Our patients are expected to be long suffering in more ways than one. Where choices exist they are almost invariably made by professionals. We provide little information about choices in treatment, about technical quality, or about the outcome of our care, partly because we do not believe that the patient should know, and partly because we do not ourselves have the information. The more closely we examine our practice the more clear it becomes that we do not have good information about the efficacy of many of the treatments we offer. We have little information about cost and a tendency to believe that it should not be our concern. The idea that the professional (doctor, nurse, etc) knows best and "will do the best for the patient" is both widely held and ill founded.

Failure to come to terms with fundamental change in health care provision might also be included in the list of outmoded concepts. Health care professionals tend to think of themselves in isolation, providing service in a one to one direct relationship with the patient, while the reality is that health care is increasingly a complex and sophisticated process comparable with relatively sophisticated manufacturing processes. It demands the successful integration of the work of large numbers of individual professionals to achieve a successful outcome. Apparently simple processes are in reality complex. The example of the fifty plus steps entailed in obtaining a chest radiograph of a hospital inpatient which require coordinated activity of more than twelve people is becoming well known. Yet in considering provision of health care we concentrate on individual steps or the activity of individual professionals and neglect the integration of activity which is the key to successful delivery of care. The term "management" is widely used in the health service, but we give little consideration to the overall management of the processes of health care at a time when its increasing complexity makes this ever more important.

\section{Components of quality}

How are we to change this situation and provide the better quality of health care that we all wish? We must first have a common concept of the components that comprise quality.

Acceptability - The service that we offer must be one that is acceptable to both patients and staff. The needs of the patients must be seen as pre-eminent, but the service must also be acceptable to the staff who provide it.

Promptness - Our services should be available with a minimum of delay. It is not clear what that minimum should be nor what the consequences of achieving it are, but we must set standards and work towards them. As we do so the effects should be clearly evaluated. Can we cope with the change in practice that is needed to deliver prompt service? Is there any health gain? Do costs increase?

Relevance - The service we provide must meet the needs of the patients rather than our own needs as health care professionals.

Information - We must provide patients with information both about what treatment comprises and its likely outcome, and this information must be provided in a form which the patient can use.

Respect for patient choice - The patient must be given adequate information on which to base decisions about treatment, and we must respect these decisions, both when we believe our knowledge is adequate to offer a clear choice and when, increasingly, we are becoming aware that we do not have that knowledge.

Technical quality - We must strive to achieve the highest possible technical quality and to improve our methods of measurement so that we can be confident about outcome. As know- 
ledge improves our information to patients and their ability to choose will improve.

Cost effectiveness - We must accept the need to deliver a cost effective service which gives good value for money.

All of these will necessitate re-evaluation of many of our habits and customs. We will have to define the service required and ensure that it is provided in the most appropriate form, by the most appropriate individual, and in the most effective fashion. We will probably have to change much of what we do. That brings me to what I see as the key to improving quality of health care - namely, the management of the overall process of care.

Approaches to the management of health care with a view to improving quality have in general been fragmented. The nursing profession has been heavily committed, but its work has tended not to involve other professions. Doctors have come relatively late to formal approaches to improving health care. Medical audit initially was regarded as a private matter. Only recently has the concept of clinical audit involving other professions gained wider acceptance. There have been a few broader based approaches to quality issues. Confidential inquiries into maternal and perinatal mortality and into perioperative death are conspicuous examples, but they are external and depend on uncertain local action for implementation of their recommendations. The Audit Commission has taken a lead on several issues, but its conclusions again lack local ownership and depend on an ill developed local management structure for implementation. Valuable as these approaches are, I do not see them as the way forward. We need to include all of the people involved, both the professions and the other health care workers. The failures of a porter, a receptionist or a switchboard operator militate against a quality service almost as much as those of a doctor or a nurse. Clinical audit may lead to some improvement in standards of care but its outcomes have often been disappointing, and unless we can achieve a means by which they are structurally incorporated in our management of patients they will remain so. The findings of external analysis also need to be incorporated into patient management locally. Often where the need to do so is greatest, the ability to do so is least.

\section{Formal management of patient care}

Formal structuring of patient management provides the way forward. Most of the work we undertake is routine, but perhaps because of our belief in the uniqueness of the individual and the importance of the one to one relationship between therapist and patient we structure our work as if every case were the exception rather than the rule. Because we reinvent the process each time we work inefficiently, omitting various parts of high quality care, reduplicating others, making ourselves inefficient, and lowering the quality of outcome. Sometimes we deliver care that we recognise as being below our own view of the best standards of care. If we planned our care on the basis of the routine, and considered the modification required for the exception, care could be much improved. I believe it is no coincidence that some of the most rigidly structured care is given for the most complex conditions - for example, chemotherapy regimens for diseases such as haematological malignancies - and it can be shown to be highly successful. At the cost of a little effort and a great change in attitude we could achieve great improvements for all of our patients.

Different centres are now taking these approaches to structuring of health care. The concept of the overall treatment plan, the "anticipated recovery pathway," or the management protocol is becoming more common. I believe that this is the way forward in improving health care, but it must be linked to another fundamental concept - the needs of the patient. As health care has become more complex its provision has been increasingly dominated by the needs of the professionals providing it, rather than those of the patients receiving it. In devising our protocols we must throughout relate them to the needs of the patient in every way, starting with the concept of bringing the service to the patient not the patient to the service, and accepting throughout the primacy of the patient's needs and decisions. These ideas are incorporated in the concept of the "patient focused hospital" and offer a clear route to improvement in health care.

At Central Middlesex Hospital we have accepted this concept and are working to implement it. We believe that ambulatory care will be increasingly important, and we seek to implement the patient focused approach for both ambulatory and inpatient care. For the vast majority of our patient contracts we believe that care can be managed in this way, that its delivery will be more predictable and its quality higher, and that it will offer the patients a service that is both greatly improved and much more acceptable. In structuring health care we will examine the tasks carried out and ensure that each is undertaken by the most appropriate individual professional. The role of nurses is likely to be increasingly important, and we see nurses as key individuals in managing planned care for each patient. We have started replanning our service along these lines in two surgical services: orthopaedics and urology. We believe the change will be substantially completed within two years. The effort is mainly intellectual but is considerable. The expense has been modest. I believe that our goal of a radical improvement in quality of patient service - the objective of this conference - is attainable. I do not suggest that we have a blueprint that can be applied effortlessly and in all situations. I am convinced that acceptance of the principle of moving consciously to managed patient care is crucial. In the working out of that principle there are great gains - for the care providers and, most of all, for our patients. The gains are achievable. The challenge for us is to achieve them. 